\title{
Editorial
}

\section{Revista de Enfermagem da Universidade Federal de Santa Maria celebra seus 10 anos de criação: trajetória ascendente e os novos desafios para a excelência editorial}

Nursing Journal of the Federal University of Santa Maria celebrates its 10 years: ascending trajectory and new challenges for editorial excellence

Revista de Enfermagem da Universidade Federal de Santa celebra sus 10 años de creación: trayectoria ascendente y nuevos retos para la excelencia editorial

\section{Francisco Carlos Félix Lana ${ }^{\mathrm{I}}$, Dalva Cezar da Silva ${ }^{\mathrm{II}}$}

A Biblioteca Virtual de Saúde (BVS) Enfermagem, desde sua criação, em 2005, recebeu da BIREME - Centro Latino-Americano e do Caribe de Informação em Ciências da Saúde/Organização Panamericana de Saúde (OPS)/Organização Mundial da Saúde (OMS), a responsabilidade de conduzir processos de avaliação para seleção e permanência de periódicos de Enfermagem para as bases Base de Dados de Enfermagem (BDENF) e Literatura latino-Americana e do Caribe em Ciências da Saúde (LILACS). Para tanto, foi criada uma Comissão de Avaliação composta por editores de revistas e experts em comunicação científica com a função de estabelecer os critérios de indexação nestas bases, construir fluxos e fundamentalmente, avaliar a normalização e o mérito dos periódicos que se apresentam para submissão.

\footnotetext{
${ }^{\text {I }}$ Enfermeiro. Doutor em Enfermagem pela Escola de Enfermagem de Ribeirão Preto - Universidade de São Paulo (USP). Professor Titular da Universidade Federal de Minas Gerais (UFMG), Escola de Enfermagem. Belo Horizonte, Minas Gerais, Brasil. E-mail: xicolana@ufmg.br, Orcid: https://orcid.org/0000-0001-9043-3181

II Enfermeira. Doutora em Enfermagem pela Universidade Federal do Rio Grande do Norte (UFRN). Enfermeira no Departamento de Enfermagem da Universidade Federal de Santa Maria (UFSM). Santa Maria, Rio Grande do Sul, Brasil. E-mail: dalvacezarsilva@yahoo.com.br, Orcid: https://orcid.org/0000-0003-1143-7817
} 
Revista de Enfermagem da Universidade Federal de Santa Maria celebra seus 10 anos... 2

A partir de 2010, com a expansão da Pós-Graduação em Enfermagem no Brasil, induzida pela Área de Enfermagem da Coordenação de Aperfeiçoamento de Pessoal de Nível Superior (CAPES), a BVS Enfermagem se alinha a esse processo e intensifica sua iniciativa na perspectiva de ampliar o quantitativo de periódicos indexados na BDENF e LILACS. Desta maneira, passou a acolher as inúmeras publicações que foram sendo criadas no âmbito dos programas de Pós-Graduação em Enfermagem no país. Esse movimento foi estruturado por meio de dois vetores: um de indução para criação e fortalecimento das revistas e outro, de formação no campo da comunicação científica.

A Revista de Enfermagem da Universidade Federal de Santa Maria (REUFSM) foi um dos periódicos que se apresentou neste contexto. $\mathrm{Na}$ submissão para a inclusão na BDENF, foram avaliados os fascículos: 1 e 2 do volume 1. Essa avaliação apontou algumas fragilidades relacionadas à normalização bibliográfica e descrição metodológica de alguns manuscritos. Entretanto, o parecer que aprovou a indexação, destacou que a revista estava estruturada, com processo de arbitragem bem descrito e que as temáticas eram coerentes e consistentes com os objetivos da Revista. Ressaltou o vínculo com a Pós-Graduação da Universidade Federal de Santa Maria (UFSM) e conclui a importância da Revista para a enfermagem. Enfatizou também que, mesmo estando no seu início, tinha excelente apresentação, organização e qualidade nos textos, constituindo em mais um periódico de relevância para a área, com potencial para uma ótima classificação e inclusão em bases de dados relevantes. Realçou ainda que foi criada com boa estrutura e vitalidade, podendo atingir patamares de excelência se continuar com a qualidade observada nos fascículos analisados, como também se mantiver e atualizar a boa organização e disposição das informações no site da revista. ${ }^{1}$ Portanto, naquele momento, em seus dois primeiros 
fascículos, a Revista já expressava sua qualidade científica e o seu potencial em se tornar uma revista de referência na área de Enfermagem.

Cabe ressaltar que a BVS Enfermagem instituiu um fluxo virtuoso no qual a BDENF figura como "porta de entrada” para as revistas jovens, seguida pela LILACS e também pela REV@ENF - Portal de revistas na metodologia SciELO. Foi na esteira desse fluxo que, em 2013, a Revista se apresentou para indexação na LILACS. Assim, a Comissão de Avaliação de Periódicos da BVS Enfermagem aprovou a inclusão da REUFSM, destacando em seu parecer que ficava evidente a preocupação do corpo editorial da revista em atender critérios de um periódico de maior qualificação, uma vez que se observou um salto qualitativo do início da sua publicação em 2011 para o volume atual em 2013, confirmando seu potencial para inclusão em novas bases de dados bibliográficos. Em síntese, a Revista atendeu as recomendações feitas em 2011 pela BVS Enfermagem, como também avançou na melhoria da gestão científica e técnica.

Quatro anos se passaram e foi possível verificar que a Revista não apenas manteve suas características verificadas nas avaliações para BDENF e LILACS, mas percebeu-se claramente sua trajetória ascendente. Observou-se que no período de 2011 a 2014, a Revista publicou 257 artigos, uma média de 64 artigos anuais, sendo 192 destes como originais de pesquisa e uma taxa de rejeição de 58,5\%. ${ }^{2}$ Esses indicadores representam vitalidade e uma tendência da revista em se tornar uma referência na área. A visibilidade alcançada em quatro anos de sua criação é algo para se considerar e comemorar. A explicação está nas bases estratégias de seu processo de criação e de sustentabilidade: vinculação com a pós-graduação, adesão à plataforma Sistema Eletrônico de Editoração de Revistas (SEER) e hospedagem na plataforma de periódicos eletrônicos da UFSM, sendo fundamental o apoio institucional e o fortalecimento das inúmeras parcerias. ${ }^{1-2}$ Ademais, cabe ressaltar 
Revista de Enfermagem da Universidade Federal de Santa Maria celebra seus 10 anos... 4

que também, foi muito importante a participação de seus Editores nos movimentos criados em torno da BVS Enfermagem e nos Fóruns de Editores da Associação Brasileira de Enfermagem (ABEn) e Associação Brasileira de Editores Científicos (ABEC), dentre outros.

Nos 10 anos de criação foram mais de 755 artigos publicados e observa-se uma tendência crescente. Em 2011, foram 56 artigos publicados e em 2020 - já como publicação contínua, foram 98. Destaque para que deste conjunto, foram publicados 593 originais de pesquisa, representando $78,5 \%$ do total. Isso demonstra o elevado grau de contribuição para a ciência da Enfermagem, fortalecendo diferentes linhas e agendas de pesquisa.

Desse período para os dias atuais, a Revista continuou sua trajetória ascendente e adotou as principais iniciativas para elevar o patamar de qualidade de periódicos: a publicação contínua para acelerar o processo de comunicação; redistribuição do corpo editorial para garantir representatividade científica e abrangência institucional e geográfica do corpo de editores e pareceristas; adoção do Digital Object Identifier (DOI), número reconhecido globalmente como identificador único e persistente de documentos e indexação no Directory of Open Access Journals (DOAJ) que é o índice de periódicos de acesso aberto de qualidade reconhecido globalmente.

Dessa forma, a tão almejada visibilidade passa por incluir o periódico nos diferentes processos de avaliação de qualidade da produção científica; isto é, significa indexar a revista nas principais bases de dados, o que a posicionará na direção de se constituir em referência para a comunidade científica da área. Nesse sentido, destaca-se a indexação na CUIDEN - Base de Datos Bibliográfica de La Fundación Index e que no ranking cuiden Citacion a REUFSM ocupa o 27ํㅡㄴ lugar - 2019, com o índice 0,642 , sendo que estava no $21^{\circ}$ lugar- 2018, relevância do contínuo 


\section{5 | Lana FCF, Silva DC}

monitoramento do processo de atualização na base, cabendo ao editor monitorar esse processo. Outro indicador é o Google Scholar, em que na lista das revistas tendo como referência o índice h de 2019, a REUFSM ocupa a 96 ${ }^{\mathbf{a}}$ posição com Índice h5 de 15 e Mediana h5 19. Em relação à SCOPUS, observa-se que a Revista obteve 273 citações entre 2013 e 2020, sendo 67,7\% na área de Enfermagem e 18,5\% na área da Medicina. Esse resultado é bastante promissor para uma Revista que ainda não está indexada nessa base. Recomenda-se à REUFSM se preparar para alcançar novas bases de dados, tais como a SciELO, a SCOPUS e a Web of Science.

Além desses avanços consideráveis, novos desafios se impõem à Revista de Enfermagem da UFSM e também às demais revistas de Enfermagem. Todos eles se relacionam para a visibilidade e o desenvolvimento de novas estratégias para acelerar o impacto científico. Pode-se destacar: ${ }^{3-4}$

a) Redução dos tempos entre submissão, aceite e publicação (fast track).

b) Adoção e permanente atualização das boas práticas editoriais e de integridade científica com adoção das diretrizes éticas para revisores do Committee on Publication Ethics (COPE).

c) Aumento do rigor teórico-metodológico - adesão aos consensos internacionais e requisitos fundamentais para os diferentes tipos de estudo.

d) Manter atualizado seu corpo editorial de alto nível e capaz de prospectar bons artigos.

e) Adesão à ciência aberta e seus princípios incluindo a transparência e abertura progressiva da revisão por pares, como também de dados de pesquisa em repositórios - Transparency and Openness Promotion.

f) Alta seletividade no processo de revisão, mesmo que isso implique na publicação de um número pequeno de artigos. 
Revista de Enfermagem da Universidade Federal de Santa Maria celebra seus 10 anos... 6

g) Adotar padrões internacionais de editoração.

h) Participar de redes sociais e de pesquisadores.

Neste sentido, um determinante para a superação dos desafios apontados está na gestão editorial: quadro de editores, apoio institucional, técnico, financeiro e político. Destaca-se que é essencial o fortalecimento do projeto político da Revista, no sentido de ampliar a qualificação dos editores; profissionalizar a editoração, ampliar a participação da equipe editorial em cursos, conferências, eventos na área da comunicação científica; assim como, a organização de uma equipe robusta para que este trabalho se profissionalize.

Por fim, reafirmamos nossa avaliação de que a jovem Revista de Enfermagem da UFSM trilhou em seus 10 anos de existência um caminho sustentável do ponto de vista científico, houve avanços importantes e que os novos desafios que se impõem possam ser superados para que a Revista continue avançando e desta forma, contribua para a expansão e o fortalecimento da ciência da Enfermagem.

\section{Referências}

1. Budó MLD, Beck CLC, Silva DC, Prestes FC, Soares RSA, Garcia RP. História da Revista de Enfermagem da Universidade Federal de Santa Maria. Rev Gaúcha Enferm. 2016;37(4):e64924. doi: http://dx.doi.org/10.1590/1983-1447.2016.04.64924.

2. Budó MLD, Beck CLC, Silva DC. Caracterização das Publicações da Revista de Enfermagem da Universidade Federal de Santa Maria - REUFSM. In: VIII WEC - Workshop de Editoração Científica; 2014 nov 10-13; Campos do Jordão. São Paulo: Associação Brasileira de Editores Científicos - ABEC Brasil; 2014 [acesso em 2020 dez 06]. Disponível em: http://ocs.abecbrasil.org.br/index.php/WEC/viiiwec/paper/viewFile/75/77

3. SciELO. Critérios, política e procedimentos para a admissão e a permanência de periódicos na Coleção SciELO Brasil. Maio 2020. [acesso em 2020 dez 06]. Disponível em: https://wp.scielo.org/wp-content/uploads/20200500-Criterios-SciELO-Brasil.pdf 
4. Marques F. Táticas para elevar o impacto. [acesso em 2020 dez 06]. Disponível em: https://revistapesquisa.fapesp.br/taticas-para-elevar-o-impacto/

Editora Científica Chefe: Cristiane Cardoso de Paula

Editora Científica: Tânia Solange Bosi de Souza Magnago

\section{Autor correspondente}

Francisco Carlos Félix Lana

E-mail: xicolana@ufmg.br

Endereço: Av. Alfredo Balena, 190 - Campus Saúde - Universidade Federal de Minas Gerais (UFMG). Belo Horizonte, Minas Gerais - Brasil. CEP: 30130-100

\section{Contribuições de Autoria}

\section{1 - Francisco Carlos Félix Lana}

Concepção ou desenho do estudo/pesquisa; análise e/ou interpretação dos dados; revisão final com participação crítica e intelectual no manuscrito.

\section{2 - Dalva Cezar da Silva}

Análise e/ou interpretação dos dados; revisão final com participação crítica e intelectual no manuscrito.

\section{Como citar este artigo}

Lana FCF, Silva DC. Revista de Enfermagem da Universidade Federal de Santa Maria celebra seus 10 anos de criação: trajetória ascendente e os novos desafios para a excelência editorial. Rev. Enferm. UFSM. 2020 [Acesso em: Ano Mês Dia];vol.10 e1: 1-7. DOI:https://doi.org/10.5902/2179769263704 